\title{
Distribution of integers that are sums of three squares of primes
}

\author{
by \\ JiANYA LIU and TAO ZHAN (Jinan)
}

1. Introduction. In 1938, Hua [7] proved that almost all integers $n$ satisfying some necessary conditions are sums of three squares of primes:

$$
n=p_{1}^{2}+p_{2}^{2}+p_{3}^{2} \text {. }
$$

To be more precise, let

$$
\mathfrak{H}=\{n \geq 1: n \equiv 3(\bmod 24), n \not \equiv 0(\bmod 5)\},
$$

and let $E(N)$ be the number of $n \in \mathfrak{H}$ not exceeding $N$ that cannot be represented as (1.1). Then Hua's result actually states that $E(N) \ll N \log ^{-A} N$ for some positive constant $A$. Later Schwarz [16] proved that Hua's estimate holds for arbitrary $A>0$. And in 1993, Leung and Liu [10] improved the upper bound of $E(N)$ to $N^{1-\delta}$, where $\delta>0$ is some computable absolute constant depending on, among other things, the constants in the DeuringHeilbronn phenomenon. Recently, Bauer, Liu, and Zhan [1] have dealt with this problem via a different approach without the Deuring-Heilbronn phenomenon, and established $E(N) \ll N^{77 / 80+\varepsilon}$, where $\varepsilon>0$ is arbitrary.

In this paper, we make the following improvement.

Theorem 1. Let $N \geq 2$, and $E(N)$ as above. Then for any $\theta>47 / 50$ we have

$$
E(N) \ll N^{\theta} .
$$

We prove our Theorem 1 by the circle method. The main difficulty arises in treating the enlarged major arcs, and it is overcome in Theorem 2 in Section 2, whose proof forms the bulk of the paper, Sections $2-5$.

2000 Mathematics Subject Classification: 11P32, 11P05, 11N36, 11P55.

The first author supported by The National Natural Science Foundation (Grant \#10041004) and the Trans-Century Training Programme Foundation for the Talents by the Ministry of Education.

The second author supported by The National Natural Science Foundation. 
To get our improvement in Theorem 1 (also in Theorem 2) we use, in addition to the aforementioned approach of [1], some observation of Liu and Liu [11]. Here, as in [1] and [11], the possible existence of a Siegel zero does not have special influence, hence the Deuring-Heilbronn phenomenon can be avoided. The key point is that there are three prime variables in our problem, and we can take advantage of this by saving the factor $r_{0}^{-1 / 2+\varepsilon}$ in Lemma 2.1 below. With this saving, our enlarged major arcs can be treated by the large sieve inequality, Gallagher's lemma, and classical results on the distribution of zeros of $L$-functions (see Lemmas 2.2-2.5). The novelties described above not only give better results (note that Theorem 2 holds with $P=N^{3 / 25-\varepsilon}$ ), but also lead us to a technically simpler proof.

We conclude this introduction by mentioning that the distribution in short intervals of integers $n$ that can be represented as (1.1) has been studied by the authors [12] and Mikawa [13].

Notation. As usual, $\varphi(n), \mu(n)$, and $\Lambda(n)$ stand for the functions of Euler, Möbius, and von Mangoldt respectively, $d(n)$ is the divisor function, and $d_{\nu}(n)$ is the generalized divisor function which is defined as the number of representations of $n$ as a product of $\nu$ positive integers. We use $\chi \bmod q$ and $\chi^{0} \bmod q$ to denote a Dirichlet character and the principal character modulo $q$, and $L(s, \chi)$ is the Dirichlet $L$-function. For integers $a, b, \ldots$ we denote by $[a, b, \ldots]$ their least common multiple. $N$ is a large integer, and $L=\log N$. And $r \sim R$ means $R<r \leq 2 R$. If there is no ambiguity, we express $\frac{a}{b}+\theta$ as $a / b+\theta$ or $\theta+a / b$. The same convention will be applied for quotients. The letter $\varepsilon$ denotes a positive constant which is arbitrarily small.

2. The circle method and preliminaries. In order to apply the circle method, we set

$$
P=N^{3 / 25-\varepsilon}, \quad Q=N /\left(P L^{23}\right) .
$$

By Dirichlet's lemma on rational approximation, each $\alpha \in[1 / Q, 1+1 / Q]$ may be written in the form

$$
\alpha=a / q+\lambda, \quad|\lambda| \leq 1 /(q Q)
$$

for some integers $a, q$ with $1 \leq a \leq q \leq Q$ and $(a, q)=1$. We denote by $\mathfrak{M}(a, q)$ the set of $\alpha$ satisfying (2.2), and define the major arcs $\mathfrak{M}$ and the minor arcs $\mathfrak{m}$ as follows:

$$
\mathfrak{M}=\bigcup_{q \leq P} \bigcup_{\substack{a=1 \\(a, q)=1}}^{q} \mathfrak{M}(a, q), \quad \mathfrak{m}=[1 / Q, 1+1 / Q] \backslash \mathfrak{M} .
$$

It follows from $2 P \leq Q$ that the major $\operatorname{arcs} \mathfrak{M}(a, q)$ are mutually disjoint. 
For $\chi \bmod q$, define

$$
C(\chi, a)=\sum_{h=1}^{q} \bar{\chi}(h) e\left(\frac{a h^{2}}{q}\right), \quad C(q, a)=C\left(\chi^{0}, a\right) .
$$

If $\chi_{1}, \chi_{2}, \chi_{3}$ are characters $\bmod q$, then we write

$$
B\left(n, q, \chi_{1}, \chi_{2}, \chi_{3}\right)=\sum_{\substack{a=1 \\(a, q)=1}}^{q} e\left(-\frac{a n}{q}\right) C\left(\chi_{1}, a\right) C\left(\chi_{2}, a\right) C\left(\chi_{3}, a\right),
$$

and

$$
B(n, q)=B\left(n, q, \chi^{0}, \chi^{0}, \chi^{0}\right), \quad \mathfrak{S}(n, P)=\sum_{q \leq P} \frac{B(n, q)}{\varphi^{3}(q)} .
$$

Our Theorem 1 is a consequence of the following

Theorem 2. Let $\mathfrak{M}$ be as in (2.3) with $P$ determined by (2.1), and for $M=N L^{-8}$ let

$$
S(\alpha)=\sum_{M<p^{2} \leq N}(\log p) e\left(p^{2} \alpha\right) .
$$

Then for $N / 2<n \leq N$, we have

$$
\int_{\mathfrak{M}} S^{3}(\alpha) e(-n \alpha) d \alpha=\frac{\pi}{4} \mathfrak{S}(n, P) n^{1 / 2}+O\left(N^{1 / 2} L^{-4}\right) .
$$

We will prove Theorem 2 in Sections 3-5, where we will need the following preliminaries.

Lemma 2.1. Let $\chi_{j} \bmod r_{j}$ with $j=1,2,3$ be primitive characters, $r_{0}=\left[r_{1}, r_{2}, r_{3}\right]$, and $\chi^{0}$ the principal character $\bmod q$. Then

$$
\sum_{\substack{q \leq x \\ r_{0} \mid q}} \frac{1}{\varphi^{3}(q)}\left|B\left(n, q, \chi_{1} \chi^{0}, \chi_{2} \chi^{0}, \chi_{3} \chi^{0}\right)\right| \ll r_{0}^{-1 / 2+\varepsilon} \log ^{30} x .
$$

Proof. This is implied in Leung and Liu [10], so we may be brief. By the proof of Lemma 6.7 of [10], the quantity under consideration is

$$
\ll \sum_{u \mid \sigma} \frac{\left|B\left(n, u r_{0}, \chi_{1} \chi^{0}, \chi_{2} \chi^{0}, \chi_{3} \chi^{0}\right)\right|}{\varphi^{3}\left(u r_{0}\right)} \sum_{\substack{\left.q \leq x / u r_{0}\right) \\\left(q, r_{0}\right)=1}} \frac{|B(n, q)|}{\varphi^{3}(q)},
$$

where $\chi^{0}$ is the principal character modulo $u r_{0}$, and

$$
\sigma= \begin{cases}1 & \text { if } 2 \nmid r_{0} \\ 2 & \text { if } 4 \mid r_{0} \\ 4 & \text { if } 2 \| r_{0}\end{cases}
$$


By (2.5) and Vinogradov's bound

$$
|C(\chi, a)| \leq q^{1 / 2} d(q), \quad \chi \bmod q, \quad(q, a)=1
$$

we have

$$
\sum_{u \mid \sigma} \frac{\left|B\left(n, u r_{0}, \chi_{1} \chi^{0}, \chi_{2} \chi^{0}, \chi_{3} \chi^{0}\right)\right|}{\varphi^{3}\left(u r_{0}\right)} \ll \sum_{u \mid \sigma}\left(u r_{0}\right)^{-1 / 2+\varepsilon} \ll r_{0}^{-1 / 2+\varepsilon} .
$$

By Lemma $6.3(\mathrm{c})$ of $[10]$ we have $|B(n, p)| / \varphi^{3}(p)<30 / p$ for all prime $p$, and consequently,

$$
\sum_{\substack{q \leq x /\left(u r_{0}\right) \\\left(q, r_{0}\right)=1}} \frac{|B(n, q)|}{\varphi^{3}(q)} \ll \sum_{q \leq x} \frac{|B(n, q)|}{\varphi^{3}(q)} \ll \prod_{p \leq x}\left(1+\frac{30}{p}\right) \ll \log ^{30} x .
$$

Collecting the above estimates, we get Lemma 2.1 .

Lemma 2.2. Let $P \geq 2$ and $T \geq 2$, and $k=0$ or 1 . Then

$$
\sum_{q \leq P} \sum_{\chi \bmod }^{*} \int_{-T}^{T}\left|L^{(k)}\left(\frac{1}{2}+i t, \chi\right)\right|^{4} d t \ll P^{2} T \log ^{4(k+1)}\left(P^{2} T\right) .
$$

Here and in what follows, the sum $\sum^{*}$ is over all primitive characters.

Lemma 2.3. Let $P \geq 2, T \geq 2$, and $a_{m}$ with $m=1,2, \ldots$ be a sequence of complex numbers. Then

$$
\sum_{q \leq P} \sum_{\chi \bmod }^{*} \int_{q}^{T}\left|\sum_{m=M_{0}}^{M_{0}+M} \frac{a_{m} \chi(m)}{m^{i t}}\right|^{2} d t \ll \sum_{m=M_{0}}^{M_{0}+M}\left(P^{2} T+m\right)\left|a_{m}\right|^{2} .
$$

Lemma 2.4. For $T \geq 2$, let $N^{*}(\alpha, q, T)$ denote the number of zeros of all the $L$-functions $L(s, \chi)$ with primitive characters $\chi \bmod q$ in the region $\operatorname{Re} s \geq \alpha,|\operatorname{Im} s| \leq T$. Then

$$
N^{*}(\alpha, q, T) \ll(q T)^{12(1-\alpha) / 5} \log ^{c}(q T) .
$$

Lemma 2.5. Let $T \geq 2$. There is an absolute constant $c_{2}>0$ such that $\prod_{\chi \bmod q} L(s, \chi)$ is zero-free in the region

$$
\operatorname{Re} s \geq 1-c_{2} / \max \left\{\log q, \log ^{4 / 5} T\right\}, \quad|\operatorname{Im} s| \leq T,
$$

except the possible Siegel zero.

Lemmas 2.2-2.5 are well known results in number theory. For the proofs of Lemmas 2.2-2.4, see for example pp. 640 and 642, 634, and 669 in Pan and Pan [14]. For Lemma 2.2, see also Bombieri [2], and for a slightly weaker form of Lemma 2.4 which suffices for our purposes, see Huxley [9]. For the proof of Lemma 2.5, see Satz VIII.6.2 in Prachar [15]. 
3. An explicit expression. The purpose of this section is to establish an explicit expression for the left-hand side of (2.8) (see Lemma 3.1 below). In Sections $4-5$ we shall estimate this explicit expression to obtain (2.8). Define

$$
\begin{aligned}
V(\lambda) & =\sum_{M<m^{2} \leq N} e\left(m^{2} \lambda\right), \\
W(\chi, \lambda) & =\sum_{M<p^{2} \leq N}(\log p) \chi(p) e\left(p^{2} \lambda\right)-\delta_{\chi} \sum_{M<m^{2} \leq N} e\left(m^{2} \lambda\right),
\end{aligned}
$$

where $\delta_{\chi}=1$ or 0 according as $\chi$ is principal or not. Also, define

$$
\begin{aligned}
& J=\sum_{r \leq P} r^{-1 / 6+\varepsilon} \sum_{\chi \bmod r}^{*} \max _{|\lambda| \leq 1 /(r Q)}|W(\chi, \lambda)|, \\
& K=\sum_{r \leq P} r^{-1 / 6+\varepsilon} \sum_{\chi \bmod r}^{*}\left(\int_{-1 /(r Q)}^{1 /(r Q)}|W(\chi, \lambda)|^{2} d \lambda\right)^{1 / 2} .
\end{aligned}
$$

Now we state the main result of this section.

Lemma 3.1. Let $n, \mathfrak{M}$ be as in Theorem 2. Then

$$
\begin{aligned}
\int_{\mathfrak{M}} S^{3}(\alpha) & (-n \alpha) d \alpha \\
= & \frac{\pi}{4} \mathfrak{S}(n, P) n^{1 / 2}+O\left\{\left(J K^{2}+J K+J\right) L^{34}\right\}+O\left(N^{1 / 2} L^{-4}\right),
\end{aligned}
$$

where $\mathfrak{S}(n, P)$ is as in $(2.6)$.

Proof. Introducing Dirichlet characters, we can rewrite the exponential sum $S(\alpha)$ as (see for example [3], Section 26, (2))

$$
S\left(\frac{a}{q}+\lambda\right)=\frac{C(q, a)}{\varphi(q)} V(\lambda)+\frac{1}{\varphi(q)} \sum_{\chi \bmod q} C(\chi, a) W(\chi, \lambda) .
$$

Thus,

$$
\int_{\mathfrak{M}} S^{3}(\alpha) e(-n \alpha) d \alpha=I_{0}+3 I_{1}+3 I_{2}+I_{3}
$$

where

$$
\begin{aligned}
I_{j}= & \sum_{q \leq P} \frac{1}{\varphi^{3}(q)} \sum_{\substack{a=1 \\
(a, q)=1}}^{q} C^{3-j}(q, a) e\left(-\frac{a n}{q}\right) \int_{-1 /(q Q)}^{1 /(q Q)} V^{3-j}(\lambda) \\
& \times\left\{\sum_{\chi \bmod q} C(\chi, a) W(\chi, \lambda)\right\}^{j} e(-n \lambda) d \lambda .
\end{aligned}
$$

We will prove that $I_{0}$ gives the main term, and $I_{1}, I_{2}, I_{3}$ the error term. 
We begin with $I_{3}$, the most complicated one. Reducing the characters in $I_{3}$ into primitive characters, we have

$$
\begin{aligned}
\left|I_{3}\right|= & \mid \sum_{q \leq P} \sum_{\chi_{1} \bmod } \sum_{\chi_{2} \bmod q} \sum_{\chi_{3} \bmod q} \frac{B\left(n, q, \chi_{1}, \chi_{2}, \chi_{3}\right)}{\varphi^{3}(q)} \\
& \times \int_{-1 /(q Q)}^{1 /(q Q)} W\left(\chi_{1}, \lambda\right) W\left(\chi_{2}, \lambda\right) W\left(\chi_{3}, \lambda\right) e(-n \lambda) d \lambda \mid \\
\leq & \sum_{r_{1} \leq P} \sum_{r_{2} \leq P} \sum_{r_{3} \leq P} \sum_{\chi_{1} \bmod r_{1}}^{*} \sum_{\chi_{2} \bmod r_{2}}^{*} \sum_{\chi_{3} \bmod r_{3}}^{*} \sum_{\substack{q \leq P \\
r_{0} \mid q}} \\
& \times \frac{\left|B\left(n, q, \chi_{1} \chi^{0}, \chi_{2} \chi^{0}, \chi_{3} \chi^{0}\right)\right|}{\varphi^{3}(q)} \\
& \times \int_{-1 /(q Q)}^{1 /(q Q)}\left|W\left(\chi_{1} \chi^{0}, \lambda\right)\right| \cdot\left|W\left(\chi_{2} \chi^{0}, \lambda\right)\right| \cdot\left|W\left(\chi_{3} \chi^{0}, \lambda\right)\right| d \lambda,
\end{aligned}
$$

where $\chi^{0}$ is the principal character modulo $q$ and $r_{0}=\left[r_{1}, r_{2}, r_{3}\right]$. For $q \leq P$ and $M<p^{2} \leq N$, we have $(q, p)=1$. By (3.1), we then have $W\left(\chi_{j} \chi^{0}, \lambda\right)=$ $W\left(\chi_{j}, \lambda\right)$ for the primitive characters $\chi_{j}$ above. Using this and Lemma 2.1, we obtain

$$
\begin{aligned}
\left|I_{3}\right| \leq & \sum_{r_{1} \leq P} \sum_{r_{2} \leq P} \sum_{r_{3} \leq P} \sum_{\chi_{1} \bmod r_{1}}^{*} \sum_{\chi_{2} \bmod r_{2}}^{*} \sum_{\chi_{3} \bmod r_{3}}^{*} \int_{-1 /\left(r_{0} Q\right)}^{1 /\left(r_{0} Q\right)}\left|W\left(\chi_{1}, \lambda\right)\right| \\
& \times\left|W\left(\chi_{2}, \lambda\right)\right| \cdot\left|W\left(\chi_{3}, \lambda\right)\right| d \lambda \sum_{q \leq P} \frac{\left|B\left(n, q, \chi_{1} \chi^{0}, \chi_{2} \chi^{0}, \chi_{3} \chi^{0}\right)\right|}{\varphi^{3}(q)} \\
\ll & L^{30} \sum_{r_{1} \leq P} \sum_{r_{2} \leq P} \sum_{r_{3} \leq P} r_{0}^{-1 / 2+\varepsilon} \sum_{\chi_{1} \bmod r_{1}}^{*} \sum_{\chi_{2} \bmod r_{2}}^{*} \sum_{\chi_{3} \bmod r_{3}}^{*} \\
& \times \int_{-1 /\left(r_{0} Q\right)}^{1 /\left(r_{0} Q\right)}\left|W\left(\chi_{1}, \lambda\right)\right| \cdot\left|W\left(\chi_{2}, \lambda\right)\right| \cdot\left|W\left(\chi_{3}, \lambda\right)\right| d \lambda .
\end{aligned}
$$

If we apply the inequality

$$
r_{0}^{-1 / 2+\varepsilon} \leq r_{1}^{-1 / 6+\varepsilon} r_{2}^{-1 / 6+\varepsilon} r_{3}^{-1 / 6+\varepsilon}
$$

to the above quantity and use Cauchy's inequality, then we get 


$$
\begin{aligned}
\left|I_{3}\right| \ll & L^{30}\left\{\sum_{r_{1} \leq P} r_{1}^{-1 / 6+\varepsilon} \sum_{\chi_{1} \bmod r_{1}}^{*} \max _{|\lambda| \leq 1 /\left(r_{1} Q\right)}\left|W\left(\chi_{1}, \lambda\right)\right|\right\} \\
& \times\left\{\sum_{r_{2} \leq P} r_{2}^{-1 / 6+\varepsilon} \sum_{\chi_{2} \bmod r_{2}}^{*}\left(\int_{-1 /\left(r_{2} Q\right)}^{1 /\left(r_{2} Q\right)}\left|W\left(\chi_{2}, \lambda\right)\right|^{2} d \lambda\right)^{1 / 2}\right\} \\
& \times\left\{\sum_{r_{3} \leq P} r_{3}^{-1 / 6+\varepsilon} \sum_{\chi_{3} \bmod r_{3}}^{*}\left(\int_{-1 /\left(r_{3} Q\right)}^{1 /\left(r_{3} Q\right)}\left|W\left(\chi_{3}, \lambda\right)\right|^{2} d \lambda\right)^{1 / 2}\right\} \\
= & J K^{2} L^{30} .
\end{aligned}
$$

Similarly, we can bound $I_{2}$ and $I_{1}$ in terms of $J$ and $K$, to get

$$
\left|I_{2}\right|+\left|I_{1}\right| \ll L^{30}\left\{J K\left(\int_{-1 / Q}^{1 / Q}|V(\lambda)|^{2} d \lambda\right)^{1 / 2}+J \int_{-1 / Q}^{1 / Q}|V(\lambda)|^{2} d \lambda\right\} .
$$

By partial summation,

$$
\begin{aligned}
V(\lambda) & =\int_{M^{1 / 2}}^{N^{1 / 2}} e\left(\lambda u^{2}\right) d u+O(1+|\lambda| N) \\
& =\frac{1}{2} \sum_{M<m \leq N} m^{-1 / 2} e(m \lambda)+O(1+|\lambda| N) .
\end{aligned}
$$

Using this and the elementary estimate

$$
\sum_{M<m \leq N} m^{-1 / 2} e(m \lambda) \ll \min \left(N^{1 / 2}, M^{-1 / 2}|\lambda|^{-1}\right),
$$

one easily gets

$$
\int_{-1 / Q}^{1 / Q}|V(\lambda)|^{2} d \lambda \ll \int_{-1 / Q}^{1 / Q}\left\{\min \left(N, M^{-1}|\lambda|^{-2}\right)+(1+|\lambda| N)^{2}\right\} d \lambda \ll L^{4} .
$$

It thus follows from (3.5) and (3.6) that

$$
\left|I_{3}\right|+\left|I_{2}\right|+\left|I_{1}\right| \ll\left\{J K^{2}+J K+J\right\} L^{34} .
$$

It remains to compute $I_{0}$. Substituting (3.7) into $I_{0}$, we have

$$
\begin{aligned}
I_{0}= & \frac{1}{8} \sum_{q \leq P} \frac{B(n, q)}{\varphi^{3}(q)} \int_{-1 /(q Q)}^{1 /(q Q)}\left\{\sum_{M<m \leq N} m^{-1 / 2} e(m \lambda)\right\}^{3} e(-n \lambda) d \lambda \\
& +O\left\{\sum_{q \leq P} \frac{|B(n, q)|}{\varphi^{3}(q)} \int_{-1 /(q Q)}^{1 /(q Q)}\left|\sum_{M<m \leq N} m^{-1 / 2} e(m \lambda)\right|^{2} d \lambda\right\} .
\end{aligned}
$$


By (3.8) and Lemma 2.1 with $r_{0}=1$, the $O$-term in (3.10) can be estimated as

$$
\ll \sum_{q \leq P} \frac{|B(n, q)|}{\varphi^{3}(q)}\left\{\int_{0}^{1 / \sqrt{M N}} N d \lambda+\int_{1 / \sqrt{M N}}^{\infty} M^{-1}|\lambda|^{-2} d \lambda\right\} \ll L^{34} .
$$

Now we extend the integral in the main term of $(3.10)$ to $[-1 / 2,1 / 2]$; by a similar argument we see that the resulting error is

$$
\ll L^{30} \int_{1 /(P Q)}^{1 / 2} M^{-3 / 2}|\lambda|^{-3} d \lambda \ll M^{-3 / 2}(P Q)^{2} L^{30} \ll N^{1 / 2} L^{-4},
$$

where we have used (2.1). Thus the main term of (3.10) becomes

$$
\frac{1}{8} \sum_{q \leq P} \frac{B(n, q)}{\varphi^{3}(q)} \sum_{\substack{M<m_{1}, m_{2}, m_{3} \leq N \\ m_{1}+m_{2}+m_{3}=n}}\left(m_{1} m_{2} m_{3}\right)^{-1 / 2}+O\left(N^{1 / 2} L^{-4}\right) .
$$

By (2.6), the first sum above is $\mathfrak{S}(n, P)$. The second sum can be calculated as

$$
\begin{aligned}
& =\sum_{\substack{1 \leq m_{1}, m_{2}, m_{3} \leq N \\
m_{1}+m_{2}+m_{3}=n}}\left(m_{1} m_{2} m_{3}\right)^{-1 / 2}+O\left(M^{1 / 2}\right) \\
& =\frac{\Gamma^{3}(1 / 2)}{\Gamma(3 / 2)} n^{1 / 2}\left\{1+O\left(n^{-1 / 2}\right)\right\}+O\left(N^{1 / 2} L^{-4}\right) \\
& =2 \pi n^{1 / 2}+O\left(N^{1 / 2} L^{-4}\right),
\end{aligned}
$$

on appealing to Lemmas 7.17 and 7.18 of Hua [8]. Thus (3.10) becomes

$$
I_{0}=\frac{\pi}{4} \mathfrak{S}(n, P) n^{1 / 2}+O\left(N^{1 / 2} L^{-4}\right) .
$$

Lemma 3.1 now follows from (3.3), (3.9), and (3.11).

4. Estimation of $J$. We have

$$
J \ll L \max _{R \leq P} J_{R}
$$

where $J_{R}$ is defined similarly to $J$ except that the sum is over $r \sim R$. The estimation of $J_{R}$ falls naturally into two cases according as $R$ is small or large. For $R>L^{B}$, where $B$ is some positive constant, one appeals to contour integration, mean-value estimates for the Dirichlet $L$-functions or their derivatives, the large sieve inequality, and Heath-Brown's identity. While for $R \leq L^{B}$, one uses the classical zero-density estimates and zero-free region for the Dirichlet $L$-functions.

We first establish the following result for large $R$. In Lemma 4.5 we shall consider small $R$. 
Lemma 4.1. Let $A>0$ be arbitrary. Then there exists a constant $B=$ $B(A)>0$ such that when $L^{B}<R \leq P$,

$$
J_{R} \ll N^{1 / 2} L^{-A},
$$

where the implied constant depends at most on $A$.

To prove this result, it suffices to show that

$$
\sum_{r \sim R} \sum_{\chi \bmod r}^{*} \max _{|\lambda| \leq 1 /(r Q)}|W(\chi, \lambda)| \ll R^{1 / 6-\varepsilon} N^{1 / 2} L^{-A}
$$

holds for $L^{B}<R \leq P$ and arbitrary $A>0$. Let

$$
\widehat{W}(\chi, \lambda)=\sum_{M<m^{2} \leq N}\left(\Lambda(m) \chi(m)-\delta_{\chi}\right) e\left(m^{2} \lambda\right) .
$$

Then

$$
W(\chi, \lambda)-\widehat{W}(\chi, \lambda)=-\sum_{j \geq 2} \sum_{M<p^{2 j} \leq N}(\log p) \chi(p) e\left(p^{2 j} \lambda\right) \ll N^{1 / 4} .
$$

Thus (4.1) is a consequence of the estimate

$$
\sum_{r \sim R} \sum_{\chi \bmod r}^{*} \max _{|\lambda| \leq 1 /(r Q)}|\widehat{W}(\chi, \lambda)| \ll R^{1 / 6-\varepsilon} N^{1 / 2} L^{-A},
$$

where $R \leq P$ and $A>0$ is arbitrary.

Let $M^{1 / 2}<u \leq N^{1 / 2}$, and let $M_{1}, \ldots, M_{10}$ be positive integers such that

$$
2^{-10} M^{1 / 2} \leq M_{1} \ldots M_{10}<u \text { and } 2 M_{6}, \ldots, 2 M_{10} \leq u^{1 / 5} .
$$

For $j=1, \ldots, 10$ let

$$
a_{j}(m)= \begin{cases}\log m & \text { if } j=1 \\ 1 & \text { if } j=2,3,4,5 \\ \mu(m) & \text { if } j=6,7,8,9,10\end{cases}
$$

We define the following functions of a complex variable $s$ :

$$
f_{j}(s)=f_{j}(s, \chi)=\sum_{m \sim M_{j}} \frac{a_{j}(m) \chi(m)}{m^{s}}, \quad F(s)=F(s, \chi)=f_{1}(s) \ldots f_{10}(s) .
$$

Now we recall Heath-Brown's identity (see Lemma 1 in [6]) for $k=5$ :

$$
\frac{\zeta^{\prime}}{\zeta}(s)=\sum_{j=1}^{5}\left(\begin{array}{l}
5 \\
j
\end{array}\right)(-1)^{j-1} \zeta^{\prime}(s) \zeta^{j-1}(s) G^{j}(s)+\frac{\zeta^{\prime}}{\zeta}(s)(1-\zeta(s) G(s))^{5},
$$

where $\zeta(s)$ is the Riemann zeta-function, and $G(s)=\sum_{m<u^{1 / 5}} \mu(m) m^{-s}$. The reason why we choose $k=5$ is that the identity with $k \leq 4$ will give weaker results, and when $k \geq 6$ it produces the same estimate as in the case 
$k=5$. Equating coefficients of the Dirichlet series on both sides provides an identity for $-\Lambda(m)$. Also, for $m \leq u$ the coefficient of $m^{-s}$ in

$$
-\frac{\zeta^{\prime}}{\zeta}(s)(1-\zeta(s) G(s))^{5}
$$

is zero. Thus,

$$
\Lambda(m)=\sum_{j=1}^{5}\left(\begin{array}{l}
5 \\
j
\end{array}\right)(-1)^{j-1} \sum_{\substack{m_{1} \ldots m_{2 j}=m \\
m_{j+1}, \ldots, m_{2 j} \leq u}}\left(\log m_{1}\right) \mu\left(m_{j+1}\right) \ldots \mu\left(m_{2 j}\right) .
$$

Applying this identity to the sum

$$
\sum_{M^{1 / 2}<m \leq u} \Lambda(m) \chi(m)
$$

one finds that (4.6) is a linear combination of $O\left(L^{10}\right)$ terms, each of which is of the form

$$
\sigma(u ; \mathbf{M})=\sum_{\substack{m_{1} \sim M_{1} \\ M^{1 / 2}<m_{1} \ldots m_{10} \leq u}} \ldots \sum_{\substack{m_{10} \sim M_{10} \\ m_{10}}} a_{1}\left(m_{1}\right) \chi\left(m_{1}\right) \ldots a_{10}\left(m_{10}\right) \chi\left(m_{10}\right)
$$

where $\mathbf{M}$ denotes the vector $\left(M_{1}, \ldots, M_{10}\right)$. By using Perron's summation formula (see for example, Lemma 3.12 of [17] or Theorem 2, p. 98 of [14]) and then shifting the contour to the left, the above $\sigma(u ; \mathbf{M})$ is

$$
\begin{aligned}
& =\frac{1}{2 \pi i} \int_{1+1 / L-i T}^{1+1 / L+i T} F(s, \chi) \frac{u^{s}-M^{s / 2}}{s} d s+O\left(\frac{N^{1 / 2} L^{2}}{T}\right) \\
& =\frac{1}{2 \pi i}\left\{\int_{1+1 / L-i T}^{1 / 2-i T}+\int_{1 / 2-i T}^{1 / 2+i T}+\int_{1 / 2+i T}^{1+1 / L+i T}\right\}+O\left(\frac{N^{1 / 2} L^{2}}{T}\right),
\end{aligned}
$$

where $T$ is a parameter satisfying $2 \leq T \leq N^{1 / 2}$. The integral on the two horizontal segments above can be easily estimated as

$$
\ll \max _{1 / 2 \leq \sigma \leq 1+1 / L}|F(\sigma \pm i T, \chi)| \frac{u^{\sigma}}{T} \ll \max _{1 / 2 \leq \sigma \leq 1+1 / L} N^{(1-\sigma) / 2} L \frac{u^{\sigma}}{T} \ll \frac{N^{1 / 2} L}{T}
$$

on using the trivial estimate

$$
\begin{aligned}
F(\sigma \pm i T, \chi) & \ll\left|f_{1}(\sigma \pm i T, \chi)\right| \ldots\left|f_{10}(\sigma \pm i T, \chi)\right| \\
& \ll\left(M_{1}^{1-\sigma} L\right) M_{2}^{1-\sigma} \ldots M_{10}^{1-\sigma} \ll N^{(1-\sigma) / 2} L .
\end{aligned}
$$

Thus,

$$
\sigma(u ; \mathbf{M})=\frac{1}{2 \pi} \int_{-T}^{T} F\left(\frac{1}{2}+i t, \chi\right) \frac{u^{1 / 2+i t}-M^{(1 / 2+i t) / 2}}{1 / 2+i t} d t+O\left(\frac{N^{1 / 2} L^{2}}{T}\right) .
$$


Since $R>L^{B}\left(\right.$ so $\left.\chi \neq \chi^{0}\right)$, in $(4.2)$ we have

$$
\begin{aligned}
\widehat{W}(\chi, \lambda) & =\sum_{M<m^{2} \leq N} \Lambda(m) \chi(m) e\left(m^{2} \lambda\right) \\
& =\int_{M^{1 / 2}}^{N^{1 / 2}} e\left(u^{2} \lambda\right) d\left\{\sum_{M^{1 / 2}<m \leq u} \Lambda(m) \chi(m)\right\}
\end{aligned}
$$

and consequently $\widehat{W}(\chi, \lambda)$ is a linear combination of $O\left(L^{10}\right)$ terms, each of which is of the form

$$
\begin{aligned}
\int_{M^{1 / 2}}^{N^{1 / 2}} e\left(u^{2} \lambda\right) d \sigma(u ; \mathbf{M})= & \frac{1}{2 \pi} \int_{-T}^{T} F\left(\frac{1}{2}+i t, \chi\right) \int_{M^{1 / 2}}^{N^{1 / 2}} u^{-1 / 2+i t} e\left(u^{2} \lambda\right) d u d t \\
& +O\left(\frac{N^{1 / 2} L^{2}}{T}(1+|\lambda| N)\right) .
\end{aligned}
$$

By taking $T=N^{1 / 2}$ and changing variables in the inner integral, we deduce from the above formulae that

$$
\begin{aligned}
|\widehat{W}(\chi, \lambda)| \ll & L^{10} \max _{\mathbf{M}} \mid \int_{-T}^{T} F\left(\frac{1}{2}+i t, \chi\right) \\
& \times \int_{M}^{N} v^{-3 / 4} e\left(\frac{t}{4 \pi} \log v+\lambda v\right) d v d t \mid+N^{3 / 25} L^{12},
\end{aligned}
$$

where the maximum is taken over all $\mathbf{M}=\left(M_{1}, \ldots, M_{10}\right)$. Since

$$
\frac{d}{d v}\left(\frac{t}{4 \pi} \log v+\lambda v\right)=\frac{t}{4 \pi v}+\lambda, \quad \frac{d^{2}}{d v^{2}}\left(\frac{t}{4 \pi} \log v+\lambda v\right)=-\frac{t}{4 \pi v^{2}}
$$

by Lemmas 4.4 and 4.3 of [17], the inner integral in (4.7) can be estimated as

$$
\begin{aligned}
& \ll M^{-3 / 4} \min \left\{\frac{N}{(|t|+1)^{1 / 2}}, \frac{N}{\min _{M<v}|t+4 \pi \lambda v|}\right\} \\
& \ll \begin{cases}N^{1 / 4} L^{2} /(|t|+1)^{1 / 2} & \text { if }|t| \leq T_{0}, \\
N^{1 / 4} L^{2} /|t| & \text { if } T_{0}<|t| \leq T,\end{cases}
\end{aligned}
$$

where $T_{0}=8 \pi N /(R Q)$. Here the choice of $T_{0}$ is to ensure that $|t+4 \pi \lambda v|>$ $|t| / 2$ whenever $|t|>T_{0}$; in fact,

$$
|t+4 \pi \lambda v| \geq|t|-4 \pi|v| /(r Q)>|t| / 2+T_{0} / 2-4 \pi N /(R Q) \geq|t| / 2 .
$$


It therefore follows from (4.7) and (4.8) that the lemma (more precisely, the $\ll$ in (4.4)) is a consequence of the following two estimates: For $0<T_{1} \leq T_{0}$, we have

$$
\sum_{r \sim R} \sum_{\chi \bmod r}^{*} \int_{T_{1}}^{2 T_{1}}\left|F\left(\frac{1}{2}+i t, \chi\right)\right| d t \ll R^{1 / 6-\varepsilon} N^{1 / 4}\left(T_{1}+1\right)^{1 / 2} L^{-A},
$$

while for $T_{0}<T_{2} \leq T$, we have

$$
\sum_{r \sim R} \sum_{\chi \bmod r}^{*} \int_{T_{2}}^{2 T_{2}}\left|F\left(\frac{1}{2}+i t, \chi\right)\right| d t \ll R^{1 / 6-\varepsilon} N^{1 / 4} T_{2} L^{-A} .
$$

Both (4.9) and (4.10) are deduced from the following bound.

Lemma 4.2. Let $F(s, \chi)$ be defined as above. Then for any $R \geq 1$ and $T_{3}>0$,

$$
\begin{aligned}
\sum_{r \sim R} \sum_{\chi \bmod r}^{*} \int_{T_{3}}^{2 T_{3}}\left|F\left(\frac{1}{2}+i t, \chi\right)\right| d t & \\
& \ll\left(R^{2} T_{3}+R T_{3}^{1 / 2} N^{3 / 20}+N^{1 / 4}\right) L^{c} .
\end{aligned}
$$

Now we can complete the proof of Lemma 4.1 immediately.

Proof of Lemma 4.1. By taking $T_{3}=T_{1}$ in Lemma 4.2, the left-hand side of (4.9) is now

$$
\ll\left(R^{2} T_{1}+R T_{1}^{1 / 2} N^{3 / 20}+N^{1 / 4}\right) L^{c} \ll R^{1 / 6-\varepsilon} N^{1 / 4}\left(T_{1}+1\right)^{1 / 2} L^{-A},
$$

provided that $L^{B}<R \leq P=N^{3 / 25-\varepsilon}$ with a sufficiently large $B$. Here $L^{B}<R$ guarantees that $N^{1 / 4} L^{c}$ is dominated by the quantity on the righthand side. This establishes (4.9). Similarly we can prove (4.10) by taking $T_{3}=T_{2}$ in Lemma 4.2. Lemma 4.1 now follows.

Lemma 4.2 has been established in Section 5 in [11]; here we provide its proof for completeness. Actually it will follow from the two propositions below.

Proposition 4.3. If there exist $M_{i}$ and $M_{j}$ with $1 \leq i<j \leq 5$ such that $M_{i} M_{j}>N^{1 / 5}$, then (4.11) is true.

Proof. Without loss of generality, we may suppose that $i=1$ and $j=2$. Using Perron's summation formula and then shifting the path of integration to the left as before, we get 


$$
\begin{aligned}
f_{1}\left(\frac{1}{2}+i t, \chi\right)= & \frac{1}{2 \pi i} \int_{1 / 2+1 / L-i N}^{1 / 2+1 / L+i N} L^{\prime}\left(\frac{1}{2}+i t+w, \chi\right) \frac{\left(2 M_{1}\right)^{w}-M_{1}^{w}}{w} d w \\
& +O\left(L^{2}\right) \\
= & \frac{1}{2 \pi i}\left\{\int_{1 / 2+1 / L-i N}^{-i N}+\int_{-i N}^{i N}+\int_{i N}^{1 / 2+1 / L+i N}\right\}+O\left(L^{2}\right) .
\end{aligned}
$$

Here one notes that the function $\left(\left(2 M_{1}\right)^{w}-M_{1}^{w}\right) / w$ has a removable singularity at $w=0$. Thus, on the above vertical segment from $-i N$ to $i N$, we have

$$
\frac{\left(2 M_{1}\right)^{w}-M_{1}^{w}}{w} \ll \frac{1}{1+|v|}
$$

where $w=u+i v$. If we use the well known bounds (see for example [14], p. 271, Exercise 6 and p. 264, (13))

$$
L^{\prime}(\sigma+i t, \chi) \ll \begin{cases}r^{(1-\sigma) / 2}|t|^{1-\sigma} \log ^{2}(r|t|) & \text { for } 0<\sigma<1,|t| \geq 2, \\ \log ^{2}(r|t|) & \text { for } \sigma \geq 1,|t| \geq 2,\end{cases}
$$

the contribution from the horizontal segments can be estimated as

$$
\begin{aligned}
& \ll \max _{0 \leq u \leq 1 / 2+1 / L} r^{(1-(1 / 2+u)) / 2}(N+|t|)^{1-(1 / 2+u)} \log ^{2}(r(N+|t|)) \frac{M_{1}^{u}}{N} \\
& \ll L^{2} \max _{0 \leq u \leq 1 / 2+1 / L} r^{1 / 6-u / 2} N^{-1 / 2-u} M_{1}^{u} \ll L^{2} .
\end{aligned}
$$

Therefore, we have

$$
\begin{aligned}
f_{1}\left(\frac{1}{2}+i t, \chi\right) & \ll \int_{-N}^{N}\left|L^{\prime}\left(\frac{1}{2}+i t+i v, \chi\right)\right| \frac{d v}{1+|v|}+L^{2} \\
& \ll L\left\{\int_{-N}^{N}\left|L^{\prime}\left(\frac{1}{2}+i t+i v, \chi\right)\right|^{4} \frac{d v}{1+|v|}\right\}^{1 / 4}+L^{2}
\end{aligned}
$$

by Hölder's inequality. Thus,

$$
\begin{aligned}
& \sum_{r \sim R} \sum_{\chi \bmod r}^{*} \int_{T_{3}}^{2 T_{3}}\left|f_{1}\left(\frac{1}{2}+i t, \chi\right)\right|^{4} d t \\
& \ll L^{4} \sum_{r \sim R} \sum_{\chi \bmod r}^{*} \int_{T_{3}}^{2 T_{3}} d t \\
& \quad \times\left\{\int_{|v| \leq 6 T_{3}}+\int_{6 T_{3} \leq|v| \leq N}\right\}\left|L^{\prime}\left(\frac{1}{2}+i t+i v, \chi\right)\right|^{4} \frac{d v}{1+|v|}+R^{2} T_{3} L^{8} \\
& =: \Sigma_{1}+\Sigma_{2}+R^{2} T_{3} L^{8},
\end{aligned}
$$


where $\Sigma_{1}$ and $\Sigma_{2}$ denote the contributions from the two integrals within the braces respectively. Clearly,

$$
\begin{aligned}
\Sigma_{1} & =L^{4} \int_{|v| \leq 6 T_{3}} \frac{d v}{1+|v|} \sum_{r \sim R} \sum_{\chi \bmod r}^{*} \int_{T_{3}+v}^{2 T_{3}+v}\left|L^{\prime}\left(\frac{1}{2}+i w, \chi\right)\right|^{4} d w \\
& \ll L^{4} \int_{|v| \leq 6 T_{3}} \frac{d v}{1+|v|} \sum_{r \sim R} \sum_{\chi \bmod r}^{*} \int_{-8 T_{3}}^{8 T_{3}}\left|L^{\prime}\left(\frac{1}{2}+i w, \chi\right)\right|^{4} d w \\
& \ll R^{2} T_{3} L^{13}
\end{aligned}
$$

on using Lemma 2.2 in the last step. To bound $\Sigma_{2}$, one first changes the order of integration to get

$$
\Sigma_{2}=L^{4} \int_{T_{3}}^{2 T_{3}} d t \sum_{r \sim R} \sum_{\chi \bmod r}^{*} \int_{6 T_{3} \leq|w-t| \leq N}\left|L^{\prime}\left(\frac{1}{2}+i w, \chi\right)\right|^{4} \frac{d w}{1+|w-t|} .
$$

Now $6 T_{3} \leq|w-t| \leq N$ implies that either $6 T_{3}+t \leq w \leq N+t$ or $-N+t \leq w \leq-6 T_{3}+t$. So, on using $T_{3} \leq t \leq 2 T_{3}$, one deduces that in either case $|w-t|-|w| / 2 \geq|w| / 2-|t| \geq 0$, and this shows that $|w-t| \geq|w| / 2$. Consequently, by Lemma 2.2,

$$
\begin{aligned}
\Sigma_{2} & \ll L^{5} \int_{T_{3}}^{2 T_{3}} d t \max _{4 T_{3} \leq x \leq N+2 T_{3}} \frac{1}{x} \sum_{r \sim R} \sum_{\chi \bmod r}^{*} \int_{x}^{2 x}\left|L^{\prime}\left(\frac{1}{2}+i w, \chi\right)\right|^{4} d w \\
& \ll R^{2} T_{3} L^{13} .
\end{aligned}
$$

Collecting the above estimates for $\Sigma_{1}$ and $\Sigma_{2}$, one obtains

$$
\sum_{r \sim R} \sum_{\chi \bmod r}^{*} \int_{T_{3}}^{2 T_{3}}\left|f_{1}\left(\frac{1}{2}+i t, \chi\right)\right|^{4} d t \ll R^{2} T_{3} L^{13} .
$$

Arguing similarly, we also have

$$
\sum_{r \sim R} \sum_{\chi \bmod r}^{*} \int_{T_{3}}^{2 T_{3}}\left|f_{2}\left(\frac{1}{2}+i t, \chi\right)\right|^{4} d t \ll R^{2} T_{3} L^{13} .
$$

Since

$$
\prod_{j=3}^{10} f_{j}\left(\frac{1}{2}+i t, \chi\right)=\sum_{M_{3} \ldots M_{10}<m \leq 2^{8} M_{3} \ldots M_{10}} \frac{b(m) \chi(m)}{m^{1 / 2+i t}}
$$

with $b(m) \leq d_{8}(m)$, one has by Lemma 2.3 , 


$$
\begin{aligned}
& \sum_{r \sim R} \sum_{\chi \bmod r} \int_{T_{3}}^{*}\left|\prod_{j=3}^{2 T_{3}} f_{j}\left(\frac{1}{2}+i t, \chi\right)\right|^{2} d t \\
& \ll \sum_{M_{3} \ldots M_{10}<m \leq 2^{8} M_{3} \ldots M_{10}} \frac{\left(R^{2} T_{3}+m\right) d_{8}^{2}(m)}{m} \\
& \ll\left(R^{2} T_{3}+M_{3} \ldots M_{10}\right) L^{c} \ll\left\{R^{2} T_{3}+\frac{N^{1 / 2}}{M_{1} M_{2}}\right\} L^{c} .
\end{aligned}
$$

One thus concludes from Hölder's inequality and (4.12)-(4.14) that

$$
\begin{aligned}
\sum_{r \sim R} \sum_{\chi \bmod r}^{*} \int_{T_{3}}^{2 T_{3}} \mid F( & \left.\frac{1}{2}+i t, \chi\right) \mid d t \\
\ll & \left\{\sum_{r \sim R} \sum_{\chi \bmod r}^{*} \int_{T_{3}}^{2 T_{3}}\left|f_{1}\left(\frac{1}{2}+i t, \chi\right)\right|^{4} d t\right\}^{1 / 4} \\
& \times\left\{\sum_{r \sim R} \sum_{\chi \bmod r}^{*} \int_{T_{3}}^{2 T_{3}}\left|f_{2}\left(\frac{1}{2}+i t, \chi\right)\right|^{4} d t\right\}^{1 / 4} \\
& \times\left\{\sum_{r \sim R} \sum_{\chi \bmod r}^{*} \int_{T_{3}}^{2 T_{3}}\left|\prod_{j=3}^{10} f_{j}\left(\frac{1}{2}+i t, \chi\right)\right|^{2} d t\right\}^{1 / 2} \\
\ll & \left(R^{2} T_{3}\right)^{1 / 2}\left\{R^{2} T_{3}+\frac{N^{1 / 2}}{M_{1} M_{2}}\right\}^{1 / 2} L^{c} \\
\ll & \left(R^{2} T_{3}+R T_{3}^{1 / 2} N^{3 / 20}\right) L^{c},
\end{aligned}
$$

since $M_{1} M_{2}>N^{1 / 5}$. This proves Proposition 4.3.

Proposition 4.4. If there is a partition $\left\{J_{1}, J_{2}\right\}$ of the set $\{1, \ldots, 10\}$ such that

$$
\prod_{j \in J_{1}} M_{j}+\prod_{j \in J_{2}} M_{j} \ll N^{3 / 10}
$$

then (4.11) is true.

Proof. For $\nu=1,2$ define

$$
F_{\nu}(s, \chi):=\prod_{j \in J_{\nu}} f_{j}(s, \chi)=\sum_{n \ll N_{\nu}} \frac{b_{\nu}(n) \chi(n)}{n^{s}},
$$

where $N_{\nu}=\prod_{j \in J_{\nu}} M_{j}$ and $b_{\nu}(n) \ll L d_{10}(n)$. Applying Lemma 2.3 we see that 


$$
\begin{aligned}
\sum_{r \sim R} & \sum_{\chi \bmod r}^{*} \int_{T_{3}}^{2 T_{3}}\left|F\left(\frac{1}{2}+i t, \chi\right)\right| d t \\
\ll & \left\{\sum_{r \sim R} \sum_{\chi \bmod r}^{*} \int_{T_{3}}^{2 T_{3}}\left|F_{1}\left(\frac{1}{2}+i t, \chi\right)\right|^{2} d t\right\}^{1 / 2} \\
& \times\left\{\sum_{r \sim R} \sum_{\chi \bmod r}^{*} \int_{T_{3}}^{2 T_{3}}\left|F_{2}\left(\frac{1}{2}+i t, \chi\right)\right|^{2} d t\right\}^{1 / 2} \\
\ll & \left\{R^{2} T_{3}+\sum_{n \ll N_{1}}\left|b_{1}(n)\right|^{2}\right\}^{1 / 2}\left\{R^{2} T_{3}+\sum_{n \ll N_{2}}\left|b_{2}(n)\right|^{2}\right\}^{1 / 2} L^{c} \\
\ll & \left(R^{2} T_{3}+N_{1}\right)^{1 / 2}\left(R^{2} T_{3}+N_{2}\right)^{1 / 2} L^{c} \\
\ll & \left(R^{2} T_{3}+R T_{3}^{1 / 2} N^{3 / 20}+N^{1 / 4}\right) L^{c},
\end{aligned}
$$

since $N_{1}+N_{2} \ll N^{3 / 10}$, and $N_{1} N_{2} \ll N^{1 / 2}$. This proves Proposition 4.4.

Proof of Lemma 4.2. In view of Proposition 4.3, we may assume that $M_{i} M_{j} \leq N^{1 / 5}$ for all $i, j$ satisfying $1 \leq i<j \leq 5$. It follows that there is at most one $M_{j}$ with $1 \leq j \leq 5$ such that $M_{j}>N^{1 / 10}$. Without loss of generality, we can suppose this exceptional $M_{j}$ is $M_{1}$, so for $j=2,3,4,5$ we have $M_{j} \leq N^{1 / 10}$. From this and the assumption that $M_{6}, \ldots, M_{10} \leq N^{1 / 10}$, we deduce that $M_{j} \leq N^{1 / 10}$ holds for $j=2,3, \ldots, 10$.

Although $M_{1}$ may exceed $N^{1 / 10}$, it is bounded from above by the inequality $M_{1} M_{2} \leq N^{1 / 5}$. From this and the assumption $M^{1 / 2} \ll M_{1} \ldots M_{10} \ll$ $N^{1 / 2}$, we see that there is an integer $l$ with $2 \leq l \leq 8$ such that

$$
M_{1} \ldots M_{l} \leq N^{1 / 5} \text {, but } M_{1} \ldots M_{l+1}>N^{1 / 5} .
$$

Take $N_{1}=M_{1} \ldots M_{l+1}$ and $N_{2}=M_{l+2} \ldots M_{10}$. Then

$$
N^{1 / 5} \ll N_{1} \ll N^{1 / 5} M_{l+1} \ll N^{1 / 5} N^{1 / 10} \ll N^{3 / 10},
$$

and

$$
N_{2} \ll N^{1 / 2} / N_{1} \ll N^{3 / 10}
$$

Thus $N_{1}+N_{2} \ll N^{3 / 10}$, i.e., the assumption of Proposition 4.4 is satisfied. Lemma 4.2 now follows from Proposition 4.4.

Now we treat the case $R \leq L^{B}$.

Lemma 4.5. Let $A>0$ and $B>0$ be arbitrary. Then for $R \leq L^{B}$, we have

$$
J_{R} \ll N^{1 / 2} L^{-A}
$$

where the implied constant depends at most on $B$. 
Proof. We use the explicit formula (see [3], p. 109 and 120, or [14], p. 313)

$$
\sum_{m \leq u} \Lambda(m) \chi(m)=\delta_{\chi} u-\sum_{|\gamma| \leq T} \frac{u^{\varrho}}{\varrho}+O\left\{\left(\frac{u}{T}+1\right) \log ^{2}(q u T)\right\}
$$

where $\varrho=\beta+i \gamma$ is a non-trivial zero of the function $L(s, \chi)$, and $2 \leq T \leq u$ is a parameter. Taking $T=N^{1 / 6}$ in (4.16), and then inserting it into $\widehat{W}(\chi, \lambda)$, using $M^{1 / 2}<u \leq N^{1 / 2}, M=N L^{-2}$, and (2.1) we get

$$
\begin{aligned}
\widehat{W}(\chi, \lambda) & =\int_{M^{1 / 2}}^{N^{1 / 2}} e\left(u^{2} \lambda\right) d\left\{\sum_{n \leq u}\left(\Lambda(m) \chi(m)-\delta_{\chi}\right)\right\} \\
& =\int_{M^{1 / 2}}^{N^{1 / 2}} e\left(u^{2} \lambda\right) \sum_{|\gamma| \leq N^{1 / 6}} u^{\varrho-1} d u+O\left\{N^{1 / 3}(1+|\lambda| N) L^{2}\right\} \\
& \ll N^{1 / 2} L^{3} \sum_{|\gamma| \leq N^{1 / 6}} N^{(\beta-1) / 2}+O\left(N^{7 / 15}\right) .
\end{aligned}
$$

Now let $\eta(T)=c_{2} \log ^{-4 / 5} T$. By Lemma $2.5, \prod_{\chi \bmod q} L(s, \chi)$ is zerofree in the region $\sigma \geq 1-\eta(T),|t| \leq T$ except for the possible Siegel zero. But by Siegel's theorem (see for example [3], Section 21) the Siegel zero does not exist in the present situation, since $r \leq L^{B}$. Thus, by Lemma 2.4,

$$
\begin{aligned}
\sum_{|\gamma| \leq N^{1 / 6}} N^{(\beta-1) / 2} & \ll L^{c} \int_{0}^{1-\eta\left(N^{1 / 6}\right)}\left(N^{1 / 6}\right)^{12(1-\alpha) / 5} N^{(\alpha-1) / 2} d \alpha \\
& \ll L^{c} N^{-\eta\left(N^{1 / 6}\right) / 10} \ll \exp \left(-c_{3} L^{1 / 5}\right) .
\end{aligned}
$$

Consequently,

$$
\sum_{r \sim R} \sum_{\chi \bmod r}^{*} \max _{|\lambda| \leq 1 /(r Q)}|\widehat{W}(\chi, \lambda)| \ll N^{1 / 2} L^{-A},
$$

where $R \leq P$, and $A>0$ is arbitrary. Lemma 4.5 now follows from (4.17), (4.2), and (4.3).

5. Estimation of $K$. In this section, we estimate $K$ by establishing the following Lemma 5.1. We remark that in proving Lemma 5.1 we need not distinguish the two cases $R>L^{B}$ and $R \leq L^{B}$ as in Lemmas 4.1 and 4.5, since we need not save a factor $L^{-A}$ on the right-hand side of (5.1).

LEMMA 5.1. We have

$$
K \ll L^{c}
$$

where $c>0$ is some absolute constant. 
Proof. By the definition of $K$ and (4.3), we have

$$
\begin{aligned}
K & \ll L \max _{R \leq P} \sum_{r \sim R} r^{-1 / 6+\varepsilon} \sum_{\chi \bmod r}^{*}\left(\int_{-1 /(r Q)}^{1 /(r Q)}|W(\chi, \lambda)|^{2} d \lambda\right)^{1 / 2} \\
& \ll L \max _{R \leq P} \sum_{r \sim R} r^{-1 / 6+\varepsilon} \sum_{\chi \bmod r}^{*}\left(\int_{-1 /(r Q)}^{1 /(r Q)}|\widehat{W}(\chi, \lambda)|^{2} d \lambda\right)^{1 / 2}+1 .
\end{aligned}
$$

Thus to establish (5.1), it suffices to show that

$$
\sum_{r \sim R} \sum_{\chi \bmod r}^{*}\left(\int_{-1 /(r Q)}^{1 /(r Q)}|\widehat{W}(\chi, \lambda)|^{2} d \lambda\right)^{1 / 2} \ll R^{1 / 6-\varepsilon} L^{c}
$$

holds for $R \leq P$ and some $c>0$.

By Gallagher's lemma (see [4], Lemma 1), we have

$$
\begin{aligned}
\int_{-1 /(r Q)}^{1 /(r Q)}|\widehat{W}(\chi, \lambda)|^{2} d \lambda & \\
& \ll\left(\frac{1}{R Q}\right)^{2} \int_{-\infty}^{\infty}\left|\sum_{\substack{v<m^{2} \leq v+r Q \\
M<m^{2} \leq N}}\left(\Lambda(m) \chi(m)-\delta_{\chi}\right)\right|^{2} d v \\
& \ll\left(\frac{1}{R Q}\right)^{2} \int_{M-r Q}^{N}\left|\sum_{\substack{v<m^{2} \leq v+r Q \\
M<m^{2} \leq N}}\left(\Lambda(m) \chi(m)-\delta_{\chi}\right)\right|^{2} d v .
\end{aligned}
$$

Let $X=\max (v, M)$ and $Y=\min (v+r Q, N)$. Then the sum in (5.3) can be written as

$$
\sum_{X<m^{2} \leq Y}\left(\Lambda(m) \chi(m)-\delta_{\chi}\right)
$$

Using Heath-Brown's identity to this sum, and applying Perron's formula as before, we see that (5.4) is a linear combination of $O\left(L^{10}\right)$ terms, each of which has the form

$$
\sigma(u ; \mathbf{M}):=\frac{1}{2 \pi} \int_{-T}^{T} F\left(\frac{1}{2}+i t, \chi\right) \frac{Y^{(1 / 2+i t) / 2}-X^{(1 / 2+i t) / 2}}{1 / 2+i t} d t+O\left(\frac{N^{1 / 2} L^{2}}{T}\right),
$$

where $\mathbf{M}, F(s, \chi)$ are as in Section 4 , and $T$ is a parameter satisfying $2 \leq$ $T \leq N^{1 / 2}$. One easily sees that 


$$
\begin{aligned}
\frac{Y^{(1 / 2+i t) / 2}-X^{(1 / 2+i t) / 2}}{1 / 2+i t} & =\frac{1}{2} \int_{X}^{Y} u^{-3 / 4+i t / 2} d u \\
& =\frac{1}{2} \int_{X}^{Y} u^{-3 / 4} e\left(\frac{t}{4 \pi} \log u\right) d u
\end{aligned}
$$

The integral can be easily estimated as

$$
\ll Y^{1 / 4}-X^{1 / 4} \ll(v+r Q)^{1 / 4}-v^{1 / 4} \ll v^{1 / 4}\left\{(1+r Q / v)^{1 / 4}-1\right\} .
$$

Since $v$ satisfies $M-r Q \leq v \leq N$, and $r Q \leq 2 R Q \leq 2 P Q=2 N L^{-23}=$ $2 M L^{-15}$, the above quantity is $\ll v^{-3 / 4} R Q \ll M^{-3 / 4} R Q$. On the other hand, one has trivially

$$
\frac{Y^{(1 / 2+i t) / 2}-X^{(1 / 2+i t) / 2}}{1 / 2+i t} \ll \frac{Y^{1 / 4}}{|t|} \ll \frac{N^{1 / 4}}{|t|} .
$$

Collecting the two upper bounds, we get

$$
\begin{aligned}
\frac{Y^{(1 / 2+i t) / 2}-X^{(1 / 2+i t) / 2}}{1 / 2+i t} & \ll \min \left(M^{-3 / 4} R Q, \frac{N^{1 / 4}}{|t|}\right) \\
& \ll L^{2} \min \left(\frac{R Q}{N^{3 / 4}}, \frac{N^{1 / 4}}{|t|}\right) .
\end{aligned}
$$

Taking

$$
T=N^{1 / 2}, \quad T_{0}=N /(Q R),
$$

we see that

$$
\begin{aligned}
\sigma(u ; \mathbf{M}) \ll & \frac{R Q L^{2}}{N^{3 / 4}} \int_{|t| \leq T_{0}}\left|F\left(\frac{1}{2}+i t, \chi\right)\right| d t \\
& +N^{1 / 4} L^{2} \int_{T_{0}<|t| \leq T}\left|F\left(\frac{1}{2}+i t, \chi\right)\right| \frac{d t}{|t|}+O\left(L^{2}\right) .
\end{aligned}
$$

Consequently, (5.3) becomes

$$
\begin{aligned}
\int_{-1 /(r Q)}^{1 /(r Q)}|\widehat{W}(\chi, \lambda)|^{2} d \lambda \ll & N^{-1 / 2} L^{30} \max _{\mathbf{M}}\left(\int_{|t| \leq T_{0}}\left|F\left(\frac{1}{2}+i t, \chi\right)\right| d t\right)^{2} \\
& +\frac{N^{3 / 2} L^{30}}{(Q R)^{2}} \max _{\mathbf{M}}\left(\int_{T_{0}<|t| \leq T}\left|F\left(\frac{1}{2}+i t, \chi\right)\right| \frac{d t}{|t|}\right)^{2} \\
& +\frac{N L^{30}}{(Q R)^{2}} .
\end{aligned}
$$


Now the left-hand side of (5.2) is

$$
\begin{aligned}
\ll & N^{-1 / 4} L^{15} \max _{\mathbf{M}} \sum_{r \sim R} \sum_{\chi \bmod r}^{*} \int_{|t| \leq T_{0}}\left|F\left(\frac{1}{2}+i t, \chi\right)\right| d t \\
& +\frac{N^{3 / 4} L^{15}}{R Q} \max _{\mathbf{M}} \sum_{r \sim R} \sum_{\chi \bmod r}^{*} \int_{T_{0}<|t| \leq T}\left|F\left(\frac{1}{2}+i t, \chi\right)\right| \frac{d t}{|t|}+\frac{N^{1 / 2} R L^{15}}{Q} .
\end{aligned}
$$

Thus, to prove (5.2) it suffices to show that the estimate

$$
\sum_{r \sim R} \sum_{\chi \bmod r}^{*} \int_{T_{1}}^{2 T_{1}}\left|F\left(\frac{1}{2}+i t, \chi\right)\right| d t \ll R^{1 / 6-\varepsilon} N^{1 / 4} L^{c}
$$

holds for $R \leq P$ and $0<T_{1} \leq T_{0}$, and

$$
\sum_{r \sim R} \sum_{\chi \bmod r}^{*} \int_{T_{2}}^{2 T_{2}}\left|F\left(\frac{1}{2}+i t, \chi\right)\right| d t \ll R^{1 / 6-\varepsilon}(R Q) N^{-3 / 4} T_{2} L^{c}
$$

holds for $R \leq P$ and $T_{0}<T_{2} \leq T$.

The estimates (5.5) and (5.6) follow from Lemma 4.2. The proof of Lemma 5.1 is complete.

Proof of Theorem 2. By Lemmas 3.1, 4.1, 4.5, and 5.1, we get (2.8). This proves Theorem 2.

6. Proof of Theorem 1. Let $B(n, q)$ be as in (2.6), and define

$$
\Pi(n, X)=\left(1+\frac{B(n, 2)}{\varphi^{3}(2)}+\ldots+\frac{B\left(n, 2^{3}\right)}{\varphi^{3}\left(2^{3}\right)}\right) \prod_{2<p \leq X}\left(1+\frac{B(n, p)}{\varphi^{3}(p)}\right) .
$$

Then by (4.1) in Mikawa [13],

$$
\Pi(n, X) \gg \prod_{2<p \leq X}\left(1-\frac{3}{p}\right) \gg \frac{1}{\log ^{3} X}
$$

To derive Theorem 1 from Theorem 2, we need to show that $\mathfrak{S}(n, P) \gg$ $\log ^{-3} n$ for almost all $n \in \mathfrak{H} \cap(N / 2, N]$. This has been established in Lemma 6.1 (ii) of [1] by comparing $\mathfrak{S}(n, P)$ with $\Pi\left(n, P^{3 / 2}\right)$.

LEMMA 6.1. If $N^{\beta}<X \leq N^{1 / 4-\varepsilon}$ for some positive constant $\beta$, then

$$
\sum_{N / 2<n \leq N}\left|\mathfrak{S}(n, X)-\Pi\left(n, X^{3 / 2}\right)\right| \ll N^{1+\varepsilon} X^{-1 / 2} .
$$

Now we can give 
Proof of Theorem 1. Let $N$ be a sufficiently large integer, and $n \in \mathfrak{H} \cap$ $(N / 2, N]$. Let

$$
r(n)=\sum_{\substack{n=p_{1}^{2}+p_{2}^{2}+p_{3}^{2} \\ M<p_{j}^{2} \leq N}}\left(\log p_{1}\right)\left(\log p_{2}\right)\left(\log p_{3}\right) .
$$

Then

$$
r(n)=\int_{0}^{1} S^{3}(\alpha) e(-n \alpha) d \alpha=\int_{\mathfrak{M}}+\int_{\mathfrak{m}},
$$

where $\mathfrak{M}, \mathfrak{m}$, and $S(\alpha)$ are as in (2.3) and (2.7).

To estimate the contribution from the minor arcs, one notes that each $\alpha \in \mathfrak{m}$ can be written as (2.2) for some $P<q \leq Q$ and $1 \leq a \leq q$ with $(q, a)=1$. We now apply Theorem 2 of Ghosh [5], which states that, for $\alpha \in \mathfrak{m}$,

$$
S(\alpha) \ll N^{1 / 2+\varepsilon}\left(P^{-1}+N^{-1 / 4}+Q N^{-1}\right)^{1 / 4} \ll N^{1 / 2-3 / 50+2 \varepsilon} .
$$

Also, we easily derive the following mean-value estimate for $S(\alpha)$ :

$$
\int_{0}^{1}|S(\alpha)|^{4} d \alpha \ll L^{4} \sum_{\substack{m_{1}^{2}+m_{2}^{2}=m_{3}^{2}+m_{4}^{2} \\ m_{j}^{2} \leq N}} 1 \ll N^{1+\varepsilon} .
$$

It therefore follows from Bessel's inequality, (6.3), and (6.4) that

$$
\begin{aligned}
\sum_{N / 2<n \leq N}\left|\int_{\mathfrak{m}}\right|^{2} & \ll \int_{\mathfrak{m}}|S(\alpha)|^{6} d \alpha \\
& \ll\left\{\max _{\alpha \in \mathfrak{m}}|S(\alpha)|^{2}\right\} \int_{0}^{1}|S(\alpha)|^{4} d \alpha \ll N^{3-3 / 50+5 \varepsilon} .
\end{aligned}
$$

Therefore, for all $n \in \mathfrak{H} \cap(N / 2, N]$ except for a subset $\mathfrak{E}(N)$ of cardinality $O\left(N^{47 / 50+7 \varepsilon}\right)$, we have

$$
\left|\int_{\mathfrak{m}}\right| \ll N^{1-\varepsilon}
$$

The contribution from the major arcs can be handled by Theorem 2 and Lemma 6.1. By Lemma 6.1 and (6.1), for all $n \in \mathfrak{H} \cap(N / 2, N]$ except for a subset $\mathfrak{F}(N)$ of cardinality $O\left(N^{47 / 50+2 \varepsilon}\right)$, we have

$$
\mathfrak{S}(n, P) \gg \log ^{-3} n \text {. }
$$

We therefore conclude from Theorem 2, (6.2), (6.5), and (6.6) that

$$
r(n) \gg n^{1 / 2} \log ^{-3} n \quad \text { for all } n \in\{\mathfrak{H} \cap(N / 2, N]\} \backslash\{\mathfrak{E}(N) \cup \mathfrak{F}(N)\} .
$$

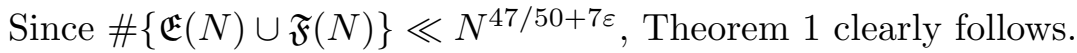




\section{References}

[1] C. Bauer, M. C. Liu and T. Zhan, On a sum of three prime squares, J. Number Theory 85 (2000), 336-359.

[2] E. Bombieri, Le grand crible dans la théorie analytique des nombres, Astérisque 18 (1974).

[3] H. Davenport, Multiplicative Number Theory, 2nd ed., Springer, Berlin, 1980.

[4] P. X. Gallagher, A large sieve density estimate near $\sigma=1$, Invent. Math. 11 (1970), 329-339.

[5] A. Ghosh, The distribution of $\alpha p^{2}$ modulo 1, Proc. London Math. Soc. (3) 42 (1981), $252-269$.

[6] D. R. Heath-Brown, Prime numbers in short intervals and a generalized Vaughan's identity, Canad. J. Math. 34 (1982), 1365-1377.

[7] L. K. Hua, Some results in the additive prime number theory, Quart. J. Math. (Oxford) 9 (1938), 68-80.

[8] -, Additive Theory of Prime Numbers, Science Press, Beijing, 1957 (in Chinese); English version: Amer. Math. Soc., Providence, RI, 1965.

[9] M. N. Huxley, Large values of Dirichlet polynomials, III, Acta Arith. 26 (1974/75), 435-444.

[10] M. C. Leung and M. C. Liu, On generalized quadratic equations in three prime variables, Monatsh. Math. 115 (1993), 133-169.

[11] J. Y. Liu and M. C. Liu, The exceptional set in the four prime squares problem, Illinois J. Math. 44 (2000), 272-293.

[12] J. Y. Liu and T. Zhan, On a theorem of Hua, Arch. Math. (Basel) 69 (1997), 375-390.

[13] H. Mikawa, On the sum of three squares of primes, in: Analytic Number Theory (Kyoto, 1996), Y. Motohashi (ed.), London Math. Soc. Lecture Notes Ser. 247, Cambridge Univ. Press, Cambridge, 1997.

[14] C. D. Pan and C. B. Pan, Fundamentals of Analytic Number Theory, Science Press, Beijing, 1991 (in Chinese).

[15] K. Prachar, Primzahlverteilung, Springer, Berlin, 1957.

[16] W. Schwarz, Zur Darstellung von Zahlen durch Summen von Primzahlpotenzen II, J. Reine Angew. Math. 206 (1961), 78-112.

[17] E. C. Titchmarsh, The Theory of the Riemann Zeta-Function, 2nd ed., University Press, Oxford, 1986.

Department of Mathematics

Shandong University

Jinan, Shandong 250100

P.R. China

E-mail: jyliu@sdu.edu.cn

zhantao@sdu.edu.cn

Received on 11. 1. 2000 